\title{
ON THE DEDEKIND SUMS AND ITS NEW RECIPROCITY FORMULA
}

\author{
XIAOYING DU AND LEI ZHANG
}

Received 08 May, 2015

\begin{abstract}
The main purpose of this paper is using the analytic method and the properties of Dirichlet $L$-functions to study the computational problem of one kind Dedekind sums, and give a new reciprocity formula for it.
\end{abstract}

2010 Mathematics Subject Classification: 11M20

Keywords: Dedekind sums, computational problem, reciprocity formula, analytic method

\section{INTRODUCTION}

Let $q$ be a natural number and $h$ an integer prime to $q$. The classical Dedekind sums

$$
S(h, q)=\sum_{a=1}^{q}\left(\left(\frac{a}{q}\right)\right)\left(\left(\frac{a h}{q}\right)\right)
$$

where

$$
((x))= \begin{cases}x-[x]-\frac{1}{2}, & \text { if } x \text { is not an integer; } \\ 0, & \text { if } x \text { is an integer, }\end{cases}
$$

describes the behaviour of the logarithm of the eta-function (see [6,7]) under modular transformations. Many authors have studied the arithmetical properties of $S(h, q)$ and obtained many interesting results, some of them can be found in [10-12]. For example, Conrey et al [3] studied the mean value distribution of $S(h, k)$, and proved the asymptotic formula

$$
\sum_{h=1}^{k}|S(h, k)|^{2 m}=f_{m}(k)\left(\frac{k}{12}\right)^{2 m}+O\left(\left(k^{\frac{9}{5}}+k^{2 m-1+\frac{1}{m+1}}\right) \cdot \ln ^{3} k\right),
$$

This work is supported by N. S. F. (11371291) and N. S. F. (11526162) and P. S. F. (2016JQ1040) of P. R. China. 
where $\sum_{h}^{\prime}$ denotes the summation over all $h$ such that $(k, h)=1$, and

$$
\sum_{m=1}^{\infty} \frac{f_{m}(n)}{n^{s}}=2 \cdot \frac{\zeta^{2}(2 m)}{\zeta(4 m)} \cdot \frac{\zeta(s+4 m-1)}{\zeta^{2}(s+2 m)} \cdot \zeta(s) .
$$

C. Jia [5] improved the error term in (1) to $O\left(k^{2 m-1} \ln ^{3} k\right)$, if $m \geq 2$.

Walum [9] obtained an identity between the mean square value of $S(h, p)$ and the fourth power mean of Dirichlet $L$-functions. That is, he proved the following:

$$
\sum_{h=1}^{p-1}|S(h, p)|^{2}=\frac{p^{2}}{\pi^{4}(p-1)} \cdot \sum_{\substack{\chi \bmod p \\ \chi(-1)=-1}}|L(1, \chi)|^{4},
$$

where $p$ be an odd prime.

Perhaps the most famous property of Dedekind sums is the reciprocity formula (see references [2,4] and [6]):

$$
S(h, k)+S(k, h)=\frac{h^{2}+k^{2}+1}{12 h k}-\frac{1}{4}
$$

for all $(h, k)=1, h>0$ and $k>0$.

An interesting three term version of (1.2) was also discovered by H. Rademacher and and E. Grosswald [7].

In this paper, as a note of [8], we use the analytic method and the properties of Dirichlet $L$-functions to study the computational problem of one kind Dedekind sums, and give a new reciprocity formula for $S(k, h)$. That is, we will prove the following:

Theorem 1. Let $h$ and $k$ are two positive odd numbers with $(k, h)=1$. Then we have the identity

$$
S(2 \cdot \bar{k}, h)+S(2 \cdot \bar{h}, k)=\frac{h^{2}+k^{2}+4}{24 h k}-\frac{1}{4},
$$

where integers $\bar{k}$ and $\bar{h}$ satisfying the congruence equation $k \cdot \bar{k} \equiv 1 \bmod h$ and $h \cdot \bar{h} \equiv$ $1 \bmod k$.

We prove this result by the analytic method and the properties of Dirichlet $L$ functions, which is distinct from other methods of proving reciprocity formula of Dedekind sums. Whether there exists a direct elementary method to prove this identity is an interesting problem.

\section{PRELIMINARIES}

To complete the proof of our theorem, we need to prove several lemmas. Hereinafter, we shall use some properties of Dirichlet $L$-functions, all of these can be found in reference [1], so they will not be repeated here. 
Lemma 1. Let $q>2$ be an integer, then for any integer $a$ with $(a, q)=1$, we have the identity

$$
S(a, q)=\frac{1}{\pi^{2} q} \sum_{d \mid q} \frac{d^{2}}{\phi(d)} \sum_{\substack{\chi \bmod d \\ \chi(-1)=-1}} \chi(a)|L(1, \chi)|^{2},
$$

where $L(1, \chi)$ denotes the Dirichlet $L$-function corresponding to the character $\chi \bmod$ $d$.

Proof. See Lemma 2 of [8].

Lemma 2. Let $a$ and $q$ are two positive odd numbers with $(a, q)=1$. Then we have the identity

$$
S(a, 2 q)+S(2 \cdot a, q)+S(\overline{2} \cdot a, q)=3 S(a, q),
$$

where $\overline{2} \cdot 2 \equiv 1 \bmod q$.

Proof. Let $\chi_{2}^{0}$ denotes the principal character mod 2. Then for any non-principal character $\chi$ mod $d$ with $(2, d)=1$, note that the identity

$$
\begin{aligned}
\left|L\left(1, \chi \chi_{2}^{0}\right)\right|^{2} & =\left|\prod_{p}\left(1-\frac{\chi(p) \chi_{2}^{0}(p)}{p}\right)^{-1}\right|^{2}=\left|\prod_{p>2}\left(1-\frac{\chi(p)}{p}\right)^{-1}\right|^{2} \\
& =\left|1-\frac{\chi(2)}{2}\right|^{2} \cdot\left|\prod_{p}\left(1-\frac{\chi(p)}{p}\right)^{-1}\right|^{2}=\left|1-\frac{\chi(2)}{2}\right|^{2} \cdot|L(1, \chi)|^{2} \\
& =\left(\frac{5}{4}-\frac{\chi(2)}{2}-\frac{\bar{\chi}(2)}{2}\right) \cdot|L(1, \chi)|^{2},
\end{aligned}
$$

from Lemma 1 and the properties of Euler function $\phi(n)$ we have

$$
\begin{aligned}
& S(a, 2 q)=\frac{1}{2 q \pi^{2}} \cdot \sum_{d \mid 2 q} \frac{d^{2}}{\phi(d)} \sum_{\substack{\chi \bmod d \\
\chi(-1)=-1}} \chi(a)|L(1, \chi)|^{2} \\
& =\frac{1}{2 q \pi^{2}} \cdot \sum_{d \mid q} \frac{d^{2}}{\phi(d)} \sum_{\substack{\chi \bmod d \\
\chi(-1)=-1}} \chi(a)|L(1, \chi)|^{2} \\
& +\frac{1}{2 q \pi^{2}} \cdot \sum_{d \mid q} \frac{4 d^{2}}{\phi(2 d)} \sum_{\substack{\chi \bmod 2 d \\
\chi(-1)=-1}} \chi(a)|L(1, \chi)|^{2} \\
& =\frac{1}{2} \cdot S(a, q)+\frac{2}{\pi^{2} q} \cdot \sum_{d \mid q} \frac{d^{2}}{\phi(d)} \sum_{\substack{\chi \bmod d \\
\chi(-1)=-1}} \chi(a) \chi_{2}^{0}(a)\left|L\left(1, \chi \chi_{2}^{0}\right)\right|^{2}
\end{aligned}
$$




$$
\begin{aligned}
= & \frac{1}{2} \cdot S(a, q) \\
& +\frac{2}{\pi^{2} q} \cdot \sum_{d \mid q} \frac{d^{2}}{\phi(d)} \sum_{\substack{\chi \bmod d \\
\chi(-1)=-1}} \chi(a)|L(1, \chi)|^{2} \cdot\left(\frac{5}{4}-\frac{\chi(2)}{2}-\frac{\bar{\chi}(2)}{2}\right) \\
= & \frac{1}{2} \cdot S(a, q)+\frac{5}{2} \cdot \frac{1}{\pi^{2} q} \cdot \sum_{d \mid q} \frac{d^{2}}{\phi(d)} \sum_{\chi \bmod d} \chi(a)|L(1, \chi)|^{2} \\
& -\frac{1}{\pi^{2} q} \cdot \sum_{d \mid q} \frac{d^{2}}{\phi(d)} \sum_{\substack{\chi \bmod d \\
\chi(-1)=-1}} \chi(2 a)|L(1, \chi)|^{2} \\
& -\frac{1}{\pi^{2} q} \cdot \sum_{d \mid q} \frac{d^{2}}{\phi(d)} \sum_{\substack{\chi \bmod d \\
\chi(-1)=-1}(\overline{2} a)|L(1, \chi)|^{2}} \\
= & \frac{1}{2} \cdot S(a, q)+\frac{5}{2} \cdot S(a, q)-S(2 \cdot a, q)-S(\overline{2} \cdot a, q) \\
= & 3 S(a, q)-S(2 \cdot a, q)-S(\overline{2} \cdot a, q)
\end{aligned}
$$

or

$$
S(a, 2 q)+S(2 \cdot a, q)+S(\overline{2} \cdot a, q)=3 S(a, q) .
$$

This proves Lemma 2.

\section{Proof of Theorem}

In this section, we shall complete the proof of our theorem. For any positive odd numbers $h$ and $k$ with $(k, h)=1$, applying Lemma 2 repeatedly we have

$$
S(k, 2 h)+S(2 \cdot k, h)+S(\overline{2} \cdot k, h)=3 S(k, h)
$$

and

$$
S(h, 2 k)+S(2 \cdot h, k)+S(\overline{2} \cdot h, k)=3 S(h, k) .
$$

Adding (3.1) and (3.2), then applying reciprocity formula (1.2) we have

$$
\begin{aligned}
S(k, 2 h)+S(2 h, k)+S(2 \cdot k, h)+S(h, 2 k)+S(\overline{2} \cdot k, h) & +S(\overline{2} \cdot h, k) \\
& =3 S(k, h)+3 S(h, k)
\end{aligned}
$$

or

$$
\begin{aligned}
\frac{4 h^{2}+k^{2}+1}{24 k h}-\frac{1}{4}+\frac{4 k^{2}+h^{2}+1}{24 k h}-\frac{1}{4}+S(\overline{2} \cdot k, h)+ & S(\overline{2} \cdot h, k) \\
& =3\left(\frac{h^{2}+k^{2}+1}{12 h k}-\frac{1}{4}\right)
\end{aligned}
$$


or

$$
S(\overline{2} \cdot k, h)+S(\overline{2} \cdot h, k)=\frac{h^{2}+k^{2}+4}{24 h k}-\frac{1}{4} .
$$

Note that if positive integers $n$ and $q$ satisfying $(n, q)=1$, then $S(n, q)=S(\bar{n}, q)$, where $\bar{n}$ satisfying the congruence equation $n \cdot \bar{n} \equiv 1 \bmod q$.

Combining this property and (3.3) we may immediately deduce the identity

$$
S(2 \cdot \bar{k}, h)+S(2 \cdot \bar{h}, k)=\frac{h^{2}+k^{2}+4}{24 h k}-\frac{1}{4} .
$$

This completes the proof of our theorem.

\section{REFERENCES}

[1] T. M. Apostol, Introduction to Analytic Number Theory. New York: Springer-Verlag, 1976. doi: 10.1007/978-3-662-28579-4.

[2] T. M. Apostol, Modular Functions and Dirichlet Series in Number Theory. New York: SpringerVerlag, 1976. doi: 10.1007/978-1-4612-0999-7.

[3] L. Carlitz, "The reciprocity theorem of Dedekind sums," Pacific J. of Math., vol. 3, pp. 513-522, 1953.

[4] J. B. Conrey, E. Fransen, R. Klein, and C. Scott, "Mean values of Dedekind sums," Journal of Number Theory, vol. 56, pp. 214-226, 1996, doi: 10.1006/jnth.1996.0014.

[5] C. Jia, "On the mean value of Dedekind sums," Journal of Number Theory, vol. 87, pp. 173-188, 2001, doi: 10.1006/jnth.2000.2580.

[6] H. Rademacher, "On the transformation of $\log \eta(\tau)$, , J. Indian Math. Soc., vol. 19, pp. 25-30, 1955.

[7] H. Rademacher and E. Grosswald, Dedekind Sums, Carus Mathematical Monographs. Washington D.C.: Math. Assoc. Amer., 1972.

[8] W. Tingting, "On the hybrid mean value of Cochrane sums and generalized Kloosterman sums," Acta Arith., vol. 153, pp. 191-197, 2012.

[9] H. Walum, “An exact formula for an average of L-series.” Ill. J. Math., vol. 26, pp. 1-3, 1982.

[10] Z. Wenpeng and L. Yanni, "A hybrid mean value related to the Dedekind sums and Kloosterman sums," Sci. China, Math., vol. 53, pp. 2543-2550, 2010.

[11] W. Zhang, "On the mean values of Dedekind sums," Journal de Theorie des Nombres, vol. 8, pp. 429-442, 1996.

[12] W. Zhang, "A note on the mean square value of the Dedekind sums," Acta Mathematica Hungarica, vol. 86, pp. 275-289, 2000.

Authors' addresses

\section{Xiaoying Du}

School of Mathematics, Jinzhong University, Jinzhong 030619, Shanxi, P. R. China

E-mail address: duxiaoying83@163.com

Lei Zhang

School of Mathematics, Jinzhong University, Jinzhong 030619, Shanxi, P. R. China

E-mail address: zhanglei840525010163.com 